
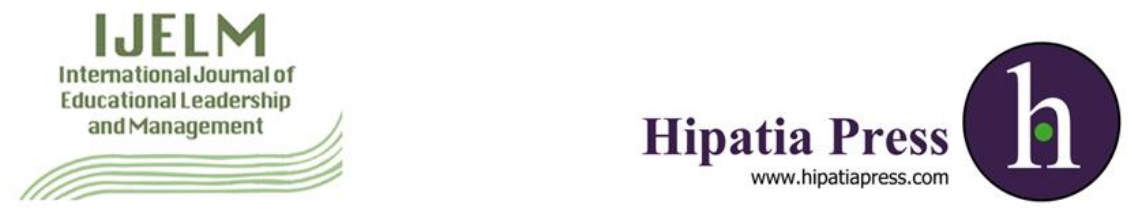

Instructions for authors, subscriptions and further details:

http://ijelm.hipatiapress.com

\title{
La Formación en el Rol de la Dirección Eficaz en la Comunidad Autónoma de Andalucía
}

Inmaculada García-Martínez ${ }^{1}$

Lina Higueras-Rodríguez ${ }^{1}$

1) Universidad de Granada. Spain

Date of publication: July $16^{\text {th }}, 2018$

Edition period: January 2018-July 2018

To cite this article: García-Martínez, I. \& Higueras-Rodríguez, L. (2018). La formación en el rol de la dirección eficaz en la comunidad autónoma de Andalucía. International Journal of Educational Leadership and

Management, 6(2), 84-109. doi: 10.17583/ijelm.2018.3103

To link this article: http://dx.doi.org/10.17583/ijelm.2018.3103

\section{PLEASE SCROLL DOWN FOR ARTICLE}

The terms and conditions of use are related to the Open Journal System and to Creative Commons Attribution License (CCAL). 


\section{Training for the Role of Effective Management in Andalusia (Spain)}

Inmaculada García-Martínez

University of Granada
Lina Higueras-Rodríguez

University of Granada

\section{Abstract}

This work has been developed around the training needs that influence the professionalization of managers. Based on international research, the impact of training on the professional performance of directors as leaders of the center has been demonstrated. Then, a look was made at the state of the training of managers in other countries, culminating in various specialized courses on the training of school leaders in Andalusia. A selection was made about the modules/courses that dealt with the theme itself: "Training on the development of the Directive Function". After carrying out the analysis, it was possible to demonstrate the need to train school leaders to carry out a leadership, capable of undertaking change processes and empowering staff and working on a common project. In turn, the head leadership is in the management and operation of the educational center.

Keywords: leadership; formation; headmaster; schools; Andalusia. 


\section{La Formación en el Rol de la Dirección Eficaz en la Comunidad Autónoma de Andalucía}

Inmaculada García-Martínez

Universidad de Granada
Lina Higueras-Rodríguez

Universidad de Granada

\section{Resumen}

El presente trabajo se ha desarrollado en torno a las necesidades formativas que influyen en una profesionalización de los directivos. A partir de investigaciones internacionales se ha evidenciado el impacto de la formación sobre el desempeño profesional de los directores como líderes del centro. A continuación, se hizo una mirada al estado de la formación de los directivos en otros países, culminando en diversos cursos especializados sobre formación de directivos escolares en la Comunidad Autónoma de Andalucía. Se hizo una selección sobre los módulos/cursos que trataban la temática en sí: "Formación sobre el desarrollo de la Función Directiva". Tras realizar el análisis se ha podido evidenciar la necesidad de formar a los directivos escolares para que lleven a cabo un liderazgo, capaz de emprender procesos de cambio y empoderar al personal y trabajar en torno a un proyecto común. A su vez, el liderazgo directivo redunda en la gestión y funcionamiento del centro educativo.

Palabras clave: liderazgo; formación; dirección escolar; escuelas; Andalucía. 


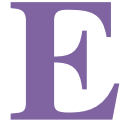

nuestros días, el liderazgo educativo se ha convertido en un elemento clave para lograr la mejora escolar (Bolívar, 2014; Moral, Amores y Ritacco, 2016). Organismos internacionales lo identifican como una línea prioritaria a seguir para lograr mejorar los resultados académicos de los estudiantes (OCDE, 2009; 2016). Con gran influencia en la investigación (Bolívar y Bolívar-Ruano, 2013; Harris, 2014, Madhlangobe y Gordon, 2012; Seashore-Louis y Wahlstrom, 2011), quienes lo señalan como el segundo factor que influye en la mejora del rendimiento académico, justo detrás del ejercicio docente (Leithwood y Louis, 2012). Todo ello ha promovido que organismos internacionales como la OCDE $(2009 ; 2016)$, hayan identificado al liderazgo pedagógico como un factor a tener en cuenta en la elaboración de las leyes educativas inter estatales. Ejemplos de ello son Chile, México o España. En el caso chileno, por ejemplo, se ha producido una reconversión del sistema escolar desde 2014. A partir de una serie de reformas, fruto de una combinación de factores sociales y políticos (Weinstein y Muñoz, 2009). Aunque las funciones de los directivos no fueron profundamente modificadas, sí que se incluyeron nuevas obligaciones adscritas a las demandas de las nuevas políticas educativas. Algunas de ellas son la obligación de desarrollar planes de mejora del centro o el diseño de itinerarios formativos para los docentes. Asimismo, se erigió un sistema de selección de directores, cuya finalidad era la de formar a directivos en materia de liderazgo para que sean capaces de movilizar y gestionar la organización educativa de forma eficaz (Campos-Vergara, Bolbarán-Ramírez, Bustos-Raggi y González-Vallejos, 2014). Algo similar ha ocurrido en México, donde se diseñaron reformas para alcanzar la mejora escolar a través de la profesionalización de la figura directiva (Morales-López, 2018). Por último, en España, algunas comunidades autónomas, especialmente Cataluña y Andalucía han desarrollado programas específicos para la formación de directores (Oliva, Toussant, López-Yáñez y Sánchez-Moreno, 2018; Silva, Del-Arco y FloresAlarcia, 2018).

Esta formación se justifica en la necesidad de mejorar los resultados escolares, una vez identificado el impacto del papel de la dirección sobre las prácticas docentes. La multiplicación de las funciones y atribuciones ligadas a los directivos, sumado a los problemas burocráticos a los que tenía que hacer frente, evidenciaron su falta de disponibilidad para hacer tales pretensiones 
184 García-Martinez \& Higueras-Rodríguez - La Formación en el Rol de la Dirección Eficaz en la Comunidad Autónoma de Andalucía

(Leithwood, Harris y Hopkins, 2008). De ahí que surgiese un punto de inflexión en la concepción del término bajo la influencia de múltiples investigaciones, avanzando desde un liderazgo burocrático a uno post burocrático (Amores, Moral y Ritacco, 2015).

En nuestros días, el liderazgo pedagógico, es decir, orientado al aprendizaje es el que más peso tiene en la enseñanza obligatoria (Bolívar, 2010). Específicamente, se buscan formas más compartidas y horizontales de liderazgo, para favorecer la implicación docente en las cuestiones educativas.

Sin embargo, pese a que cada vez es mayor el corpus investigativo relacionado con el liderazgo, en el contexto español aún persisten algunas cuestiones sin resolver. Aunque existe una tendencia creciente de estudios en España que se han centrado en la formación de directivos y su profesionalización (Barrios-Arós, Camarero-Figuerola, Tierno-García e Iranzo-García, 2015; Silva, Del-Arco y Flores-Alarcia, 2018). Hasta el momento, se ha asumido sin apenas discrepancias que el director es el que debe asumir el rol de líder, dada la posición que ostenta dentro de la organización educativa (Day, Sammons, Leithwood, Hopkins, Brown y Ahtaridou 2011), aunque siguen percibiéndose ciertas carencias profesionalizantes para llevar a cabo tal función debido, entre otras causas, a que "la exigencia de la administración educativa con los equipos directivos está aumentando día a día. La aparición de nuevos temas (...) exige que el director y el resto del equipo directivo tengan determinados conocimientos, aptitudes y capacidades para poder desarrollar su función" (VázquezToledo, Liesa-Orús y Bernal-Agudo, 2016: 159).

El eje sobre el que gira el presente trabajo son las necesidades formativas que redunden en una profesionalización de los directivos. A partir de los hallazgos investigativos alcanzados hasta el momento a nivel internacional sobre el liderazgo, se prestará atención, sobre todo, a tres grandes componentes: características y perfil de líderes (Hallinger y Heck, 2010; Harris, 2002), modalidades del liderazgo (Bush y Glover, 2003; Riveros-Barrera, 2012), sobre todo pedagógicos (Bolívar, 2010; Firestone y Martínez, 2007; Gago Rodríguez, 2003; Hallinger y Heck, 2010; Leithwood, 
2009; López-Yáñez y Lavié Martínez, 2010; Seashore-Louis et al., 2010; Poultney, 2012) y la relativa al contexto educativo, sus consecuencias y efectos sobre la organización (Fullan, 2010; Gronn, 2009; Mulford, 2006; Seashore-Louis, 2007; Spillane, 2006; Wahlstrom, 2008).

\section{Incidencia de la formación en liderazgo sobre el desarrollo profesional de la dirección}

La formación es un aspecto clave en cualquier profesión. Además, cuando la profesión está tan estrechamente entrelazada con el crecimiento y desarrollo de la sociedad, las necesidades de formación continua se incrementan exponencialmente. Tal es el caso de las profesiones ligadas al mundo educativo (Escudero, 2017).

En otros países, la dirección escolar está supeditada a un proceso formativo y evaluador que redunda en la profesionalidad de los directivos (Egido, 2013; Vaillant, 2015). Además de la pertinente formación pedagógica ligada al ejercicio docente, quienes quieren promocionar en la organización educativa, ocupando cargos de gestión, reciben una formación adicional en temas de gestión y liderazgo.

En España, desafortunadamente, la dirección no posee un marco legislativo nacional en el que se detalle el perfil de los directivos, aunque a nivel autonómico sí que se han hecho ciertos progresos para delimitar el rol de los líderes escolares. Tal es el caso de Cataluña, donde a partir de la Ley de Educación (2009) se "estableció las bases para la profesionalización de la dirección que, posteriormente, ha sido desarrollada en los Decretos 102/2010 de Autonomía de Centros y 155/2010 y 29/2015, de la dirección de los centros educativos públicos y del personal directivo profesional docente" (Silva, DelArco y Flores-Alarcia, 2018: 110). Sin embargo, pese a que la formación en liderazgo constituye un aspecto angular para una dirección eficaz (Vaillant, 2015); hasta el momento, a los aspirantes a la dirección no se les ha exigido ningún tipo de formación específica para ocupar tal puesto, más allá de presentar un proyecto afín a las líneas educativas que pretende seguir durante su mandato. 
186 García-Martinez \& Higueras-Rodríguez - La Formación en el Rol de la Dirección Eficaz en la Comunidad Autónoma de Andalucía

Si se presta atención al ámbito legislativo, se observa como la ley educativa vigente, la de Mejora de la Calidad Educativa (LOMCE, 2013), concretamente en su artículo 83, solo establece como criterio que una vez concluido el proceso de selección de los candidatos a la dirección, los candidatos deberán certificar haber superado un curso de formación sobre el desarrollo de la función directiva, impartido por el Ministerio de Educación, Cultura y Deporte o por las Administraciones educativas de las Comunidades Autónomas, si quieren participar en el concurso de méritos para la selección del director.

Al mismo tiempo, hay otros factores que deben ser tenidos en cuenta a la hora de analizar la mencionada figura y determinar su formación. En primer lugar, el acceso a la dirección es un aspecto que repercute considerablemente en la identidad profesional del directivo y en su posterior desempeño profesional (Bolívar y Ritacco, 2016). Según Montero-Alcaide (2010) la sucesión de leyes educativas ha repercutido en el modelo de dirección, experimentando éste transformaciones que van desde "la elección a la selección, pasando por la designación” (p. 417).

Otro aspecto a considerar es la temporalidad del cargo. En España, los docentes que ascienden al cargo de director lo hacen durante un tiempo limitado, viéndose obligados a volver a ejercer sus labores docentes, una vez concluido su mandato. Además, aquellos directivos designados por la Administración tienen que renovar el mandato de manera anual, lo que repercute en su estabilidad y afianzamiento como líderes escolares. Al mismo tiempo, la inestabilidad legislativa, como consecuencia, de la ausencia de un pacto educativo en nuestro país no facilita esta transición (Montero-Alcaide, 2010). Finalmente, la dualidad de funciones del director es otro factor que dificulta su desempeño, pues "la función directiva implica dos grandes facetas: funciones de gestión y funciones de liderazgo educativo" (VázquezToledo, Liesa-Orús y Bernal-Agudo, 2016, p.159).

Todo este panorama acentúa la necesidad de formación en liderazgo de los actuales directores y especialmente, de aquellos que quieran serlo en un futuro cercano. Si se pretende ir en sintonía con las investigaciones realizadas 
a nivel supranacional, que abogan por los beneficios de la implantación del liderazgo sobre las instituciones educativas, habrá que hacerlo desde el inicio del proceso, apostando por una formación en liderazgo escolar desde la formación inicial universitaria.

Al respecto, unos de los tópicos que ya se está demandando en la formación inicial de los docentes a nivel universitario, muy extendido en el panorama internacional (Campos-Vergara, Bolbarán-Ramírez, Bustos-Raggi y González-Vallejos, 2014; Morales-López, 2018), es una formación específica para la dirección escolar. Esta preparación se ha focalizado principalmente en dos ámbitos concretos: la adquisición de conocimientos, destrezas, valores y actitudes que habiliten a la dirección para liderar los procesos educativos que acontecen en los centros escolares; y el aprendizaje de ciertos elementos que le faciliten tomar decisiones y asumir responsabilidades propias de la dirección en la institución escolar (Fernández, 2006).

En esta línea la formación específica del director escolar puede concebirse como un proceso formal, intencional, consciente y dirigido, que tiene lugar dentro de un sistema interconectado. A su vez, va a conformar un conjunto de actividades organizadas, encaminadas a la adquisición y desarrollo de esos conocimientos, habilidades y valores que faciliten la adquisición y consolidación de las competencias ligadas a la dirección, es decir, aquellas competencias que capacitan al director para el desempeño adecuado de su actividad profesional directiva (Lorenzo, 2012; Valiente, González y Del Toro, 2013).

El liderazgo, en términos de modos de gestionar y dinamizar grupos de trabajo hacia el logro de unas metas comunes consensuadas (Mertkan, Arsan, Inal Cavlan y Onurkan Aliusta, 2017), guarda una relación marcada, aunque indirecta, con el logro de las metas educativas, y con la calidad y nivel académico de los alumnos. Esta relación indirecta es avalada por diversas investigaciones realizadas en el marco internacional, concluyendo que esta relación es más apreciable cuando tiene lugar en contextos socioeconómicos y socioculturales más desfavorables. Así, el liderazgo ejercido de forma eficaz por el líder de la organización, el director, así como por otros agentes 
188 García-Martinez \& Higueras-Rodríguez - La Formación en el Rol de la Dirección Eficaz en la Comunidad Autónoma de Andalucía

educativos, ayudará a mermar las posibles desigualdades sociales que puedan aparecer entre el tipo de alumnado, las existentes entre contextos de alto riesgo de absentismo y exclusión social y los de bajo riesgo y altos rendimientos, favorecerá el crecimiento y mejora de contextos desfavorecidos, etc. (ISSPP, 2017).

Como consecuencia de las múltiples tareas que son asociadas a la dirección (organizativas, pedagógicas y de gestión), se ha establecido una relación directa entre su labor y el establecimiento de la cultura imperante del personal docente (Silva, Del-Arco y Flores-Alarcia, 2018). Dicho de otro modo, la posición que ocupa el directivo en la estructura organizativa le confiere autoridad y posibilidades para crear la cultura organizativa del centro educativo (Albornoz, 2007; García, Silva-Peña y Parra, 2016).

Para cubrir las carencias formativas que poseen los directivos españoles, es necesario el diseño de un modelo de formación que haga frente a los retos y quehaceres diarios de los directores (Vázquez-Toledo, Liesa-Orús y Bernal-Agudo, 2016). En la tabla 1 se muestra la valoración de tareas de dirección educativa por directores en ejercicio dentro del contexto educativo español. Desde la visión aportada por Vázquez-Toledo et. al. en el que se muestra una valoración de las tareas de la dirección educativa y el nivel de importancia que se adquiere de cada una de ellas. Todas estas tareas son necesidades formativas que los directores escolares deben de adquirir para su desempeño profesional. A partir de estas necesidades, surge un nuevo interrogante, que ayudará a determinar y esclarecer el porqué de esta situación: ¿Cuándo sería más conveniente llevar a cabo la formación en liderazgo de los Equipos directivos? ¿Antes de que ocupen los cargos directivos, en las universidades, extendiendo la formación en liderazgo a todos los docentes? ¿O quizá sería más oportuno una vez hayan asumido el reto de la dirección? (Albornoz, 2007). 
Tabla 1.

Valoración de tareas de dirección educativa por directores en ejercicio

\section{Nivel de importancia Tareas}

\begin{tabular}{ll}
\hline Alto & $1^{\circ}$ Relaciones interpersonales \\
& $2^{\circ}$ Intervención en situaciones de conflicto de intereses \\
& entre docentes, padres, etc. \\
& $3^{\circ}$ Intervención ante problemas de disciplina de los \\
& alumnos \\
& $4^{\circ}$ Procesos de comunicación \\
& $5^{\circ}$ Organización general del centro \\
& $6^{\circ}$ Proceso de toma de decisiones \\
& $7^{\circ}$ Procesos de innovación y cambio \\
& $8^{\circ}$ Asesoramiento al profesorado \\
& $9^{\circ}$ Dirección de reuniones \\
& $10^{\circ}$ Procesos de planificación institucional \\
& $11^{\circ}$ Imagen y proyección exterior del centro \\
& $12^{\circ}$ Legislación escolar \\
& $13^{\circ}$ Apoyo al aprendizaje de los alumnos \\
& $14^{\circ}$ Gestión del tiempo para el desempeño del cargo \\
& $15^{\circ}$ Evaluación de centros y programas \\
& $16^{\circ}$ Organización de los servicios escolares \\
& $17^{\circ}$ Realización de tareas administrativas \\
\hline Bajo &
\end{tabular}

Fuente: Vázquez Toledo, Liesa Orús y Bernal Agudo (2016)

Si se realiza una reflexión en torno a las Universidades, concretamente en las Facultades de Educación, como instituciones responsables de la formación inicial de los futuros docentes y formadores, encontramos que el liderazgo, en el mejor de los casos, se halla en forma de asignaturas específicas en las distintas titulaciones de Educación. Además, se ha evidenciado que la perspectiva desde la que se enfoca no está conectada con los imperativos promovidos por los gobiernos de distintos países ni por las instituciones internacionales (OCDE, 2009), quienes apuestan por la inclusión de un liderazgo de tipo pedagógico en los centros educativos. Por otro lado, entre los planes de estudios examinados de las diferentes universidades andaluzas, no se ha encontrado ninguna asignatura que ataña específicamente a la figura de la dirección como líder escolar. Para obtener el 
190 García-Martinez \& Higueras-Rodríguez - La Formación en el Rol de la Dirección Eficaz en la Comunidad Autónoma de Andalucía

plan de estudios de cada universidad, se accedió a través de las distintas webs oficiales de las universidades (Granada, Sevilla, Huelva, Cádiz, Almería, Jaén, Córdoba y Málaga). A través de un análisis descriptivo, se obtuvo la información requerida para conocer las diferentes asignaturas de los planes de estudios analizados.

Por esta razón, una buena forma de fortalecer la figura de la dirección como líder del centro y de capacitarle para dar respuesta a los imperativos que se esperan de él, sería hacerlo desde su formación inicial, en la Universidad. De hecho, a través de su experiencia en la institución superior, los futuros docentes podrían adquirir herramientas en liderazgo, que contribuyeran a que su trabajo fuera más productivo y significativo (Gómez, 2010). En cuanto a la casuística de si debe formarse en liderazgo a todos los docentes, aunque no proyecten su carrera a la dirección, encontramos que para lograr la sostenibilidad de proyectos educativos en los centros, asentados sobre los principios de participación y donde se priorice la responsabilidad compartida en las decisiones que tienen lugar en el centro, es conveniente que todo el personal cuente con una formación suficiente, que le habilite a asumir proyectos colaborativos de tal envergadura (García, Silva-Peña y Parra, 2016; Fernández-Serrat, 2002). De hecho, ahora impera (o al menos es lo que buscan las reformas e iniciativas educativas actuales) una cultura pedagógica, fruto de un trabajo colegiado y consensuado entre el personal y la comunidad, que exige de estructuras organizativas más flexibles, para que tengan cabida la generación de cambios internos y unas condiciones óptimas para que el profesorado mejore su capital profesional. Al respecto, la universidad debe orientarse como un espacio donde se ofrezca a los profesionales de la educación aquello que necesiten para que se sientan capacitados ante los retos a los que tengan que hacer frente en su posterior desempeño profesional (Herrero, 2016).

Sin embargo, la ausencia de formación en liderazgo en las instituciones de educación superior está siendo "compensada" con cursos de formación ofertados en los Centros del Profesorado (CEP) o proyectos de investigación, catalogados dentro de la denominada formación permanente. De este modo, en los apartados siguientes se expondrá el proceso de formación 
de directores llevado a cabo en la comunidad autónoma de Andalucía, con la finalidad de hacer balance sobre los avances (de mayor o menor magnitud) que se están alcanzando.

\section{La formación de directivos escolares en liderazgo en Andalucía}

La formación de directores en los últimos años se ha erigido como una de las líneas prioritarias de la Conserjería de Educación andaluza. De hecho, desde la misma se han llevado a cabo cursos de liderazgo, localizados en los diferentes CEPs de la citada comunidad autónoma. A nivel legislativo, se encuentra que la formación de la dirección está reflejada en el Decreto 93/2013, que atañe a la formación inicial y permanente del profesorado, el artículo 14 del Sistema Andaluz de Formación Permanente del Profesorado, donde se especifica las bases de la formación de los equipos directivos y el III Plan Andaluz de Formación Permanente del Profesorado, aprobado por Orden de 31 de julio de 2014. Se trata de una formación externa que se oferta desde los CEPs y que pueden acceder cualquier director escolar.

Examinando el material existente en los diferentes cursos ofertados por la misma, se encontró uno específico "de formación sobre el desarrollo de la función directiva", distribuido en seis módulos: 1) Proyecto de Dirección; 2) Marco normativo aplicable a los centros docentes; 3) Rendición de cuentas y calidad educativa; 4) Organización y Gestión de Centros Educativos; 5) Gestión de los recursos del centro docente y 6) Factores clave para una dirección eficaz. Este curso surge en el seno de la convocatoria de la Resolución de 25 de noviembre de 2015, siguiendo las directrices establecidas en el Real Decreto 894/2014 y la Ley Orgánica 2/2006, de 3 de mayo, de Educación (LOE).

Durante la lectura de los manuales, se evidenció que todos los manuales incorporaban formación específica en liderazgo excepto el 2 y el 5 , centrándose en aspectos concretos del proceso educativo. Así, por ejemplo, aborda el liderazgo desde la perspectiva sobre cómo debe incorporarse al proyecto de dirección que los directores deben realizar. La finalidad del mismo es que los aspirantes a la dirección incorporen las competencias de liderazgo, a la hora de delimitar las líneas pedagógicas que van a llevar a cabo. 
192 García-Martinez \& Higueras-Rodríguez - La Formación en el Rol de la Dirección Eficaz en la Comunidad Autónoma de Andalucía

El módulo 3 lo alude desde la perspectiva de la evaluación del alumnado en el ámbito del liderazgo pedagógico, aportando directrices sobre cómo debe llevarse a cabo. El 4, lo relaciona con el contexto educativo, vinculándolo a las comunidades de práctica. Por su parte, el módulo 5 expone la gestión de los recursos de los centros docentes. Finalmente, el módulo 6, se ciñe a la faceta del director como líder pedagógico (Tabla 2).

Tabla 2.

Contenidos específicos de los cursos sobre liderazgo en Andalucía

\begin{tabular}{|c|c|c|}
\hline Módulo & Finalidad & Contenidos \\
\hline 1 & $\begin{array}{l}\text { Desarrollar un } \\
\text { supuesto práctico de } \\
\text { dirección }\end{array}$ & $\begin{array}{l}\checkmark \text { Proceso de selección de dirección en el } \\
\text { sistema educativo } \\
\checkmark \text { Diferentes perspectivas de autores sobre } \\
\text { proyecto de dirección } \\
\checkmark \text { Características, estructura del proyecto } \\
\text { de dirección } \\
\checkmark \text { Desarrollo de todas las actuaciones para } \\
\text { desarrollar proyecto de dirección }\end{array}$ \\
\hline 2 & $\begin{array}{l}\text { Conocer acerca del } \\
\text { Marco normativo } \\
\text { aplicable a los } \\
\text { centros docentes }\end{array}$ & $\begin{array}{l}\checkmark \text { Evaluación del sistema educativo, } \\
\text { necesidad y demanda social. } \\
\checkmark \text { El marco normativo de la rendición de } \\
\text { cuentas y evaluación institucional. } \\
\checkmark \text { La evaluación externa a través de } \\
\text { pruebas de diagnóstico } \\
\text { individualizadas. } \\
\checkmark \text { Evaluaciones y pruebas internacionales. } \\
\checkmark \text { Evaluaciones externas e internacionales } \\
\text { y la dirección escolar. }\end{array}$ \\
\hline 3 & $\begin{array}{l}\text { Conocer sobre la } \\
\text { rendición de cuentas } \\
\text { y calidad educativa }\end{array}$ & $\begin{array}{l}\checkmark \text { Sobre los conceptos: el rendimiento de } \\
\text { cuentas y la calidad educativa } \\
\checkmark \text { Índices relevantes para estimar el } \\
\text { rendimiento de cuentas y la calidad } \\
\text { educativa. El valor añadido de los } \\
\text { centros y el índice socioeconómico y } \\
\text { cultural }\end{array}$ \\
\hline
\end{tabular}




\begin{tabular}{|c|c|c|}
\hline & & $\begin{array}{l}\checkmark \text { Desempeño directivo y mejora de los } \\
\text { resultados escolares } \\
\checkmark \text { Autoevaluación institucional y plan de } \\
\text { mejora. Vinculación con el proyecto de } \\
\text { dirección y el plan de centro } \\
\checkmark \text { La evaluación del alumnado en el } \\
\text { ámbito del liderazgo pedagógico } \\
\checkmark \text { La evaluación del ejercicio directivo y } \\
\text { de las prácticas docentes } \\
\checkmark \text { La evaluación del sistema educativo } \\
\text { andaluz } \\
\checkmark \text { Otros elementos o recursos para la } \\
\text { gestión de la calidad y la mejora que } \\
\text { pueden adecuarse a los centros } \\
\text { educativos }\end{array}$ \\
\hline 4 & $\begin{array}{l}\text { Conocer la gestión y } \\
\text { organización de los } \\
\text { centros educativos }\end{array}$ & $\begin{array}{l}\checkmark \text { Los centros educativos como } \\
\text { organizaciones. Funciones y } \\
\text { responsabilidades de la dirección y del } \\
\text { equipo directivo } \\
\checkmark \text { Planificación institucional. Elaboración } \\
\text { y gestión del plan de centro } \\
\checkmark \text { La dirección de personas y grupos. La } \\
\text { cultura institucional } \\
\checkmark \text { La innovación en los centros educativos. } \\
\text { La escuela como comunidad profesional } \\
\text { de práctica. } \\
\checkmark \text { Las relaciones del centro escolar y la } \\
\text { comunidad. Participación de las familias } \\
\text { y de los diferentes sectores de la } \\
\text { comunidad. Responsabilidad e imagen } \\
\text { institucional del centro }\end{array}$ \\
\hline 5 & $\begin{array}{l}\text { Conocer la gestión } \\
\text { de recursos del } \\
\text { centro docente }\end{array}$ & $\begin{array}{ll}\checkmark & \text { La gestión de los RRHH y materiales. } \\
\checkmark & \text { La gestión administrativa y económica } \\
& \text { del centro. } \\
\checkmark & \text { Prevención de riesgos laborales. } \\
\checkmark & \text { Autoprotección. } \\
\checkmark & \text { La Innovación desde la coherencia y } \\
& \text { autonomía del centro escolar. } \\
\checkmark & \text { La informatización de la comunicación } \\
& \text { y la gestión educativa. }\end{array}$ \\
\hline
\end{tabular}


194 García-Martinez \& Higueras-Rodríguez - La Formación en el Rol de la Dirección Eficaz en la Comunidad Autónoma de Andalucía

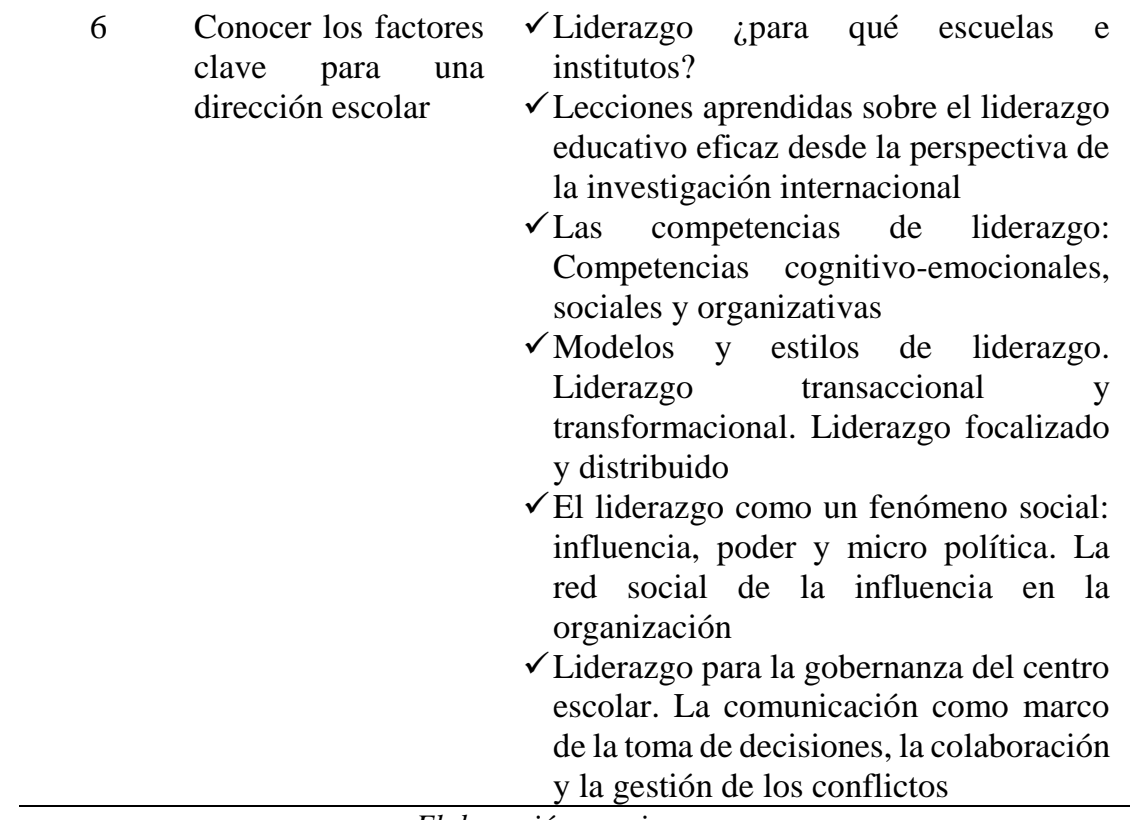

Los diferentes contenidos que se trabajan a lo largo de los diferentes módulos/cursos son esenciales para trabajar el tema de la formación de líderes educativos. Dichos contenidos han sido desarrollados con la incorporación de la Ley Orgánica para la Mejora de la Calidad Educativa (LOMCE, 2013) para dar respuesta a las demandas y necesidades que presenta los directores. Así se expone que, como requisito para poder participar en el concurso de méritos para la selección del director, deben de estar en posesión de la certificación acreditativa de haber superado un curso de formación sobre el desarrollo de la función directiva, impartido por el Ministerio de Educación, Cultura y Deporte o por las Administraciones educativas de las Comunidades Autónomas. Éstos contenidos son la base para una buena formación y posteriormente una correcta dirección escolar.

La duración mínima de los cursos es de un total de 180 horas, repartidos en 120 horas para el curso sobre Formación sobre el desarrollo de 
la función directiva, y 60 horas para el curso de Actualización de competencias directivas. Dichos cursos tienen una validez para todo el territorio nacional y una validez indefinida. Aunque para el curso de Formación de la función directiva, seré necesario un curso de actualización transcurridos 8 años desde la certificación del curso de formación. Dichos cursos podrán impartirse a distancia, mediante la utilización de sistemas telemáticos o electrónicos que garanticen la constancia de la participación y del trabajo de los participantes. En los diferentes módulos, por ello, pueden estimarse sesiones de enseñanza presencial y a distancia.

Para que se lleve a cabo la consecución de los diferentes contenidos, se proponen a lo largo de estos cursos, una serie de objetivos específicos que deben de alcanzar los usuarios. Desde por ejemplo conocer cómo se lleva a cabo un Proyecto de Dirección (PdD), proporcionando las herramientas necesarias para su ejecución y puesta en práctica, hasta conocer la gestión de cuentas y factores clave para un liderazgo eficaz (Tabla 3).

Tabla 3.

Objetivos específicos de los cursos sobre liderazgo en Andalucía

Módulo 1: - Presentar el concepto, características y finalidad de proyecto de dirección (PdD)

- Dar a conocer los factores en los que sustenta un PdD

- Llevar a cabo un supuesto práctico de un PdD

Módulo 2: - Sistematizar los conocimientos, el marco teórico y normativo en relación con la rendición de cuentas, evaluación del sistema educativo y la mejora de calidad de los centros de enseñanza.

- Adquirir herramientas y destrezas directivas en relación con el módulo.

- Reflexionar sobre la propia posible práctica directiva en relación con la evaluación y mejora del sistema educativo, de los centros y de los procesos de enseñanza y aprendizaje y adquirir las destrezas para su realización.

- Transferir al contexto de centro los conocimientos sistematizados y las herramientas adquiridas en el módulo. 
Módulo 3: - Analizar el sentido del rendimiento de cuentas y de la calidad educativa como conceptos directamente vinculados a los logros y resultados escolares del alumnado, a partir de las respuestas de los centros a las diversas necesidades educativas de estos.

- Apreciar la importancia del índice socioeconómico y cultural y del valor añadido de los centros para la valoración de los resultados que obtienen y los consiguientes planes de mejora que se adopten.

- Diseñar un plan de actuación del proyecto de dirección, a partir del plan de mejora del centro y del análisis de la realidad de partida, donde se consideren los elementos más significativos de las distintas actuaciones para el logro de los objetivos.

- Estimar algunas actuaciones directivas que se relacionan con la evaluación del alumnado desde el marco del liderazgo pedagógico.

- Apreciar la evaluación del ejercicio directivo y de las prácticas docentes desde la relevancia principal de estos dos factores para la mejora de los logros educativos del alumnado.

- Considerar el seguimiento y evaluación del proyecto de dirección como elemento para la evaluación del desempeño directivo.

- Conocer los principios, características y ámbitos de la evaluación del sistema educativo andaluz.

- Revisar elementos y recursos para la gestión de la calidad (normas, modelos, cartas de servicio) y su grado de adecuación a la organización y el funcionamiento de los centros educativos.

Módulo 4: - Comprender los centros educativos como organizaciones complejas y multidimensionales

- Conocer las perspectivas vigentes para promover, planificar y gestionar procesos de innovación educativa, cambio y desarrollo institucional

- Conocer y aplicar los marcos teóricos y esquemas de análisis necesarios para una comprensión profunda de las organizaciones educativas y de la dirección de personas y grupos en las mismas

- Favorecer la reflexión y el análisis crítico sobre problemas relevantes de la gestión de los procesos de planificación institucional, los conflictos, las relaciones del centro con la comunidad educativa y el desarrollo profesional de los docentes

Módulo 5: - Potenciar la capacidad de gestión para afrontar la dirección.

- Proporcionar a los miembros de equipos directivos formación práctica para la elaboración del proyecto de gestión y el manejo de 
aplicaciones informáticas relacionadas con la gestión administrativa y económica del centro.

- Facilitar la labor de los equipos directivos mediante el trabajo en equipo y la experiencia compartida.

- Fomentar la participación en Redes para el ejercicio de la función directiva.

Módulo 6: - Conocer los factores y aspectos que la investigación internacional asocia al liderazgo educativo eficaz.

- Identificar las competencias, los modelos y estilos asociados al liderazgo educativo y su adecuación a diferentes tipologías de centros escolares en virtud de sus características institucionales.

- Identificar el mapa de la influencia en los centros escolares: agentes intervinientes, estructuras, patrones y fuentes de poder.

- Conocer las prácticas de liderazgo frecuentemente asociadas a la mejora del aprendizaje de los alumnos y al logro de comunidades educativas inclusivas y justas.

- Conocer las prácticas de liderazgo que promueven la eficacia en la gestión y la mejora de la gobernanza del centro escolar

Fuente: Elaboración propia

Para que se lleve a cabo de manera efectiva todos estos cursos, se proponen una serie de competencias que deben de adquirir los usuarios durante el desarrollo del curso. Estas competencias dotarán confianza en sí mismo, potenciando su habilidad y capacidad de aprender, comprender y aplicar las herramientas más adecuadas en cada momento y que le permita continuar con su proceso de formación. Todas las competencias consideradas para cada curso han sido obtenidas del Anexo I del Real Decreto 894/2014, por el que se regulan las características del curso de formación sobre el desarrollo de la función directiva. A continuación, se muestran las competencias genéricas y específicas cuya adquisición debe permitir el programa formativo del curso de formación sobre el desarrollo de la función directiva. En cada una de las competencias, se expone el módulo donde se desarrollará (Tabla 4). 
198 García-Martinez \& Higueras-Rodríguez - La Formación en el Rol de la Dirección Eficaz en la Comunidad Autónoma de Andalucía

Tabla 4.

Competencias genéricas y específicas cuya adquisición debe permitir el programa formativo del curso de formación sobre el desarrollo de la función directiva.

Competencias

Módulos

\begin{tabular}{lc}
\hline $\begin{array}{l}\text { Habilidades de liderazgo y fomento del trabajo en } \\
\text { equipo. }\end{array}$ & $1,4,6$ \\
\hline Habilidades de motivación & $1,4,6$ \\
\hline $\begin{array}{l}\text { Habilidades para la gestión de la información y la toma } \\
\text { de decisiones. }\end{array}$ & $1,2,5,6$ \\
\hline Habilidades de comunicación. & $1,4,5,6$
\end{tabular}

Genéricas Habilidades para la gestión de conflictos y la 1, 4, 6 convivencia.

Habilidades para la organización, gestión y $1,3,4,5$, coordinación de un centro docente.

Habilidades de dirección estratégica: planificación, 1, 2, 3, 5 implementación y evaluación de planes y proyectos.

Habilidades de control y supervisión. 1,3,5

Habilidades para la gestión del cambio y la innovación. $\quad$ 1, 2, 4, 5,

6

Competencias

Módulos

a) Conocimiento de la planificación estratégica de 1,2 procesos.

b) Conocimiento de los estándares educativos aplicables $\quad$ 1, 2, 4 a su centro.

c) Conocimiento de los procesos internos y externos de 1,2 evaluación institucional.

d) Habilidades para implicar al profesorado en los 1,2 procesos de autonomía y rendición de cuentas

Específicas e) Habilidades para el manejo de las herramientas de 1,2 evaluación docente.

f) Actitud de fomento de la calidad continua en el centro. 1,2,5

g) Gestión de documentos profesionales $1,3,4$

h) Habilidades para la evaluación, el diseño y desarrollo $1,3,5$

de planes de mejora y fomento de la calidad del centro.

i) Dirección y gestión de los recursos humanos $1,4,6$ 
A nivel de resultados, se expone un ejemplo un programa de autoformación de líderes escolares desarrollada en el marco de un proyecto de investigación financiado por el Ministerio de Economía y Competitividad llevado a cabo por la Universidad de Sevilla (Oliva et. al, 2018). Entre los resultados obtenidos destaca que para que un grupo avance con éxito y se llegue a la reflexión crítica, la conducción de un coach o líder es esencial. Como conclusiones generales se acentúa una falta de conocimiento en profundidad del programa por parte del coach y una escasa capacidad de liderazgo. Estos elementos impiden el avance por parte del grupo para el logro de sus objetivos.

\section{Conclusiones}

En el presente artículo se ponen de manifiesto en el contexto español en cuanto a la formación de los directores para realizar las labores que se esperan de ellos, gestores y líderes escolares (Montero-Alcaide, 2010; Vázquez-Toledo, Liesa-Orús y Bernal-Agudo, 2016). Desafortunadamente, a pesar de lo expuesto, hay comunidades autónomas como Andalucía, donde ya se han llevado a cabo iniciativas contextualizadas en proyectos de investigación y reformas educativas impulsadas desde sus pertinentes conserjerías, que parecen mostrar ciertas mejoras, (Barrios-Arós et. al, 2015; Oliva et. al, 2018).

Además, aunque la universidad se considera como un espacio de formación por excelencia, aún no se ha hecho eco de la necesidad de incluir formación en liderazgo pedagógico en los planes de estudios de las titulaciones de Magisterio o Pedagogía. Este hecho implica una desaceleración en cuanto a la implantación de reformas educativas y consideraciones promovidas tanto por organismos internacionales como la OCDE, como por el propio gobierno español.

A su vez, también se demandan una mayor cantidad de iniciativas y programas que capaciten a los profesionales de la educación para ejercer mejor su labor. En primer lugar, se debería comenzar por la figura de la dirección, al situarse en la cúspide de la jerarquía educativa. En esta línea, un mayor empoderamiento de la figura directiva, junto a una mayor autonomía 
200 García-Martinez \& Higueras-Rodríguez - La Formación en el Rol de la Dirección Eficaz en la Comunidad Autónoma de Andalucía

para la implementación de iniciativas de cambio en los centros escolares, supondrían grandes avances hacia la mejora educativa. Esta mejora se vería reforzada mediante programas que fomenten el aprendizaje profesional del profesorado, a través de la creación de espacios colaborativos de aprendizaje.

En definitiva, una vez delimitada la figura de la dirección en España, nos ubicamos en la línea de investigaciones nacionales e internacionales, quienes imploran una mayor autonomía y empoderamiento de la dirección para la dinamización de procesos de cambio efectivos y beneficiosos, sobre todo, al alumnado.

\section{References}

Albornoz, C. (2007). Claves para la formación de directivos de instituciones escolares. REICE: Revista Electrónica Iberoamericana sobre Calidad, Eficacia y Cambio en Educación, 5(5), 133-138.

Amores, F. J., Moral, C. y Ritacco, M. (2015). El desarrollo de procesos de autoevaluación como capacidad del liderazgo pedagógico: Un estudio en educación secundaria en Andalucía. Revista Iberoamericana de Evaluación Educativa, 8(2), 57-76.

Barrios-Arós, C., Camarero-Figuerola, M., Tierno-García, J. M. e IranzoGarcía, P. (2015). Modelos y funciones de dirección escolar en España: el caso de Tarragona. Revista Iberoamericana de educación, 67, 89106.

Bolívar, A. (2010). ¿Cómo un liderazgo pedagógico y distribuido mejora los logros académicos? Revisión de la investigación y propuesta. Magis. Revista Internacional de Investigación en Educación, 3(5), 79-106.

Bolívar, A. y Bolívar-Ruano, M. R. (2013). Construir la capacidad de mejora escolar: liderazgo distribuido en una comunidad profesional de aprendizaje.RevistaEduc@rnos, 10,11-34.

Bolívar, A. (2014). Construcción de capacidades de la escuela: Liderazgo compartido y Comunidades Profesionales de Aprendizaje. Una propuesta de investigación. International Journal of Educational Leadership and Management, 2(2), 147-175. 
Bolívar, A. y Ritacco, M. (2016). Identidad profesional de los directores escolares en España. Un enfoque biográfico narrativo. Opción, 32(79), 163-183.

Bush, T. y Glover, D. (2003). School Leadership: Concepts and Evidence. Nottingham: National College of School Leadership.

Campos-Vergara, F., Bolbarán-Ramírez, J., Bustos-Raggi, C. y GonzálezVallejos, M. (2014). Formación de directores de excelencia: un mismo objetivo, distintas demandas. Perspectiva Educacional, Formación de Profesores, 53(2), 91-111.

Day C., Sammons P., Leithwood K., Hopkins D., Gu Q., Brown, E. y Ahtaridou E. (2011) Successful school leadership. Linking with learning and achievement. London: McGraw Hill.

Decreto 93/2013, de 27 de agosto, por el que se regula la formación inicial y permanente del profesorado en la Comunidad Autónoma de Andalucía, así como el Sistema Andaluz de Formación Permanente del Profesorado. Boletín Oficial de la Junta de Andalucía, 170, de 30 de agosto.

Egido, I. (2013). La profesionalización de la dirección escolar: tendencias internacionales. Participación educativa, 2(2), 21-28.

Escudero, J. M. (2017). La formación continua del profesorado de la educación obligatoria en el contexto español. Profesorado, Revista de Currículum y Formación del Profesorado, 21(3), 1-20.

Fernández, M. L. (2006). La formación inicial para la dirección. En Teixidó, J., Aramendi, P., Fernández, M. y Bernal, J. (2006), Simposio sobre el acceso y formación para la dirección escolar. CIOIE.

Firestone, W. y Martínez, C. (2007). Districts, teacher leaders, and distributed leadership: Changing instructional practice. Leadership and Policy in Schools, 6(1), 3-35.

Fullan, M. (2010). All Systems Go: The Change Imperative for Whole System Reform. Thousands Oaks, CA: Sage.

Gago Rodríguez, F. M. (2003). La dirección de los institutos. El liderazgo educativo que nunca existió. Organización y Gestión Educativa, 6, 1423.

García, G. G., Silva-Peña, I. y Parra, C. S. (2016). Desafíos investigativos en la formación inicial de directivos. Revista Ibero-americana de Educação, 70, 43-54. 
202 García-Martinez \& Higueras-Rodríguez - La Formación en el Rol de la Dirección Eficaz en la Comunidad Autónoma de Andalucía

Gómez, A. (2010). La formación inicial para la dirección escolar como impulso para conseguir una dirección competente en Andalucía (Tesis doctoral). Universidad de Huelva. Huelva (España).

Gronn, P. (2009). From distribute to hybrid leadership practice. En A. Harris (Ed.), Distributed leadership. Different perspectives(197-218). London: Springer.

Hallinger, P. y Heck, R. H. (2010). Collaborative leadership and school improvement: understanding the impact on school capacity and student learning. School Leadership \& Management, 30(2), 95-110.

Harris, A. (2002). Effective leadership in schools facing challenging contexts. School Leadership \& Management, 22(1), 15-26.

Harris, A. (2014). Distributed Leadership Matters: Perspectives, Practicalities, and Potential. Thousand Oaks, CA: Corwin.

Herrero, V. P. (2016). Formación de directivos escolares: Modalidad presencial versus aprendizaje on line. Opción, 32(12), 724-749.

ISSPP. International Successful school Principalship Proyect (2017). Recuperado de: https://www.uv.uio.no/ils/english/research/projects/isspp/

Leithwood, K. y Louis, K.S. (2012). Linking Leadership to Student Learning. San Francisco: Jossey-Bass.

Leithwood, K. (2009). ¿Cómo liderar nuestras escuelas? Aportes desde la investigación. Santiago de Chile: Fundación Chile.

Leithwood, K., Harris, A. y Hopkins, D. (2008). Seven strong claims about successful school leadership. School Leadership and Management, 28(1), 27-42

Ley 12/2009, de 10 de julio, de educación (LEC). Boletín Oficial del Estado, 189, 6 de agosto del 2009.

Ley Orgánica 2/2006, de 3 de mayo, de Educación (LOE). Boletín Oficial del Estado, 106, de 4 de mayo de 2006.

Ley Orgánica 8/2013, de 9 de diciembre, para la mejora de la calidad educativa (LOMCE). Boletín Oficial del Estado, 295, de 10 de diciembre de 2013.

López-Yáñez, J. y Lavié Martínez, J. M. (2008). Liderazgo y desarrollo sostenibles en las organizaciones educativas. Madrid: Ministerio de Educación y Ciencia. Dirección General de Investigación. 
Lorenzo, M. (2012). Las comunidades de liderazgo de centros educativos. Educar, 48(1), 9-21.

Fernández-Serrat, M.L. (2002). Formar para la dirección escolar: Por qué, cuándo, cómo... Profesorado, revista de currículum y formación del profesorado, 6(1), 1-12.

Madhlangobe, L. y Gordon, S. P. (2012). Culturally Responsive Leadership in a Diverse School: A Case Study of a High School Leader. NASSP Bulletin, 96(3), 177-202.

Mertkan, S., Arsan, N., Inal Cavlan, G. y Onurkan Aliusta, G. (2017). Diversity and equality in academic publishing: the case of educational leadership. Compare: A Journal of Comparative and International Education, 47(1), 46-61.

Molero, F. (2002). Cultura y liderazgo. Una relación multifacética. Boletín de Psicología, 76, 53-75.

Montero-Alcaide, A. (2016). Dirección profesional y selección de directores en el sistema educativo español. Revista española de pedagogía, 247, 417-435.

Moral, C., Amores, F. J. y Ritacco, M. (2016). Liderazgo distribuido y capacidad de mejora en centros de educación secundaria. Estudios sobre Educación, 30, 115-143.

Morales-López, G. (2015). Formación para la innovación en educación media superior. Una experiencia formativa de directores escolares en México. Consejo de redacción/Conselho de redação, 69, 229-240.

Mulford, B. (2006). Liderazgo para mejorar la calidad de la educación secundaria: algunos desarrollos internacionales. Profesorado. Revista de currículum y formación del profesorado, 10(1), 1-22.

OCDE (2009). Creating Effective Teaching and Learning Environments: First Results from TALIS. Paris, OCDE. Recuperado de: http://www.oecd.org/TALIS

OCDE (2016) Panoramas de la educación. Indicadores de la OCDE 2016. Informe español. Madrid: Ministerio de Educación, Cultura y Deporte.

Oliva, N., Toussaint, M., López-Yáñez, J. y Sánchez-Moreno, M. (2018). Análisis del desarrollo de dos grupos de autoformación de directores escolares basado en el análisis de la práctica y el coaching. En Avances en democracia y liderazgo distribuido en educación: Actas del II Congreso internacional de liderazgo y mejora de la educación. Red de Investigación sobre Liderazgo y Mejora de la Educación (RILME). 
204 García-Martinez \& Higueras-Rodríguez - La Formación en el Rol de la Dirección Eficaz en la Comunidad Autónoma de Andalucía

Orden de 31 de julio de 2014, por la que se aprueba el III Plan Andaluz de Formación Permanente del Profesorado. Boletín Oficial de la Junta de Andalucía, 170, de 2 de septiembre.

Poultney, V. (2012). The role of the effective Subject Leader: perspectives from practitioners in secondary schools. British Educational Leadership, Management and Administration Society, 21(2), 8-14.

Real Decreto 894/2014, de 17 de octubre, por el que se desarrollan las características del curso de formación sobre el desarrollo de la función directiva establecido en el artículo 134.1.c) de la Ley Orgánica 2/2006, de 3 de mayo, de Educación, así como de los correspondientes cursos de actualización de competencias directivas. Boletín Oficial del Estado, 270, de 7 de noviembre.

Resolución de 25 de noviembre de 2015, de la Dirección General de Profesorado y Gestión de Recursos Humanos, por la que se convoca curso de formación sobre el desarrollo de la función directiva establecido en el artículo 134.1 de la Ley Orgánica 2/2006, de 3 de mayo, de Educación. Boletín Oficial de la Junta de Andalucía, 233, de 01 de diciembre de 2015.

Riveros-Barrera, A. (2012) La distribución del liderazgo como estrategia de mejoramiento institucional. Educ. Educ. 15(2), 289-301.

Seashore-Louis, K. (2007). Changing the culture of schools: Professional community, organizational learning, and trust. Journal of School Leadership, 16(4), 477-489

Seashore-Louis, K., Leithwood, K., Wahlstrom, K. L. y Anderson, S. E. (2010). Learning from leadership: investigating the links to improved student learning. The Wallace Foundation Center for Applied Research and Educational Improvement/ University of Minnesota.

Seashore-Louis, K. y Wahlstrom, K. (2011). Principals as cultural leaders. Phi Delta Kappan, 92(5), 52-56.

Silva, P., Del-Arco. I. y Flores-Alarcia, O. (2018). La formación de directores escolares en Cataluña. Lecciones aprendidas a cinco años del Decreto de Dirección. Bordon. Revista de Pedagogia, 70(1), 109-124.

Spillane, J. (2006). Distributed leadership. San Francisco: Jossey-Bass.

Vaillant, D. (2015). Liderazgo escolar, evolución de políticas y prácticas y mejora de la calidad educativa. Education for All 2000-2015: 
achievements and challenges: Organización de las Naciones Unidas, pp. 1-27.

Valiente, P., González, J. y Del Toro, J. J. (2013). Fundamentos para la elaboración de una concepción teórico-metodológica de la formación especializada del director escolar. Proyecto de investigación Formación del Director Escolar. Holguín, Cuba: Universidad de Ciencias Pedagógicas José de la Luz y Caballero, Departamento de Dirección Científica Educacional.

Vázquez-Toledo, S., Liesa-Orús, M. y Bernal-Agudo, J.L. (2016). El camino hacia la profesionalización de la función directiva: el perfil competencial y la formación del director de centros educativos en España. Perfiles educativos, 38(151), 158-174.

Wahlstrom, K. L. (2008). Leadership and learning: what these articles tell us. Educational Administration Quarterly, EAQ, 44(4), 593-597.

Weinstein, J. y Muñoz, G. (2009). Calidad para todos. La reforma educacional en el punto de quiebre. Más acá de los sueños, más allá de lo posible: la concertación en chile.

Inmaculada García-Martínez. Diplomada en Magisterio especialidad en Educación Primaria. Licenciada en Psicopedagogía. Máster en Investigación e Innovación en Currículum y Formación. Actualmente, es doctoranda de la Facultad de Educación, en la Universidad de Granada, con una vinculación contractual FPU (Formación del Profesorado Universitario). Miembro del equipo de investigación FORCE (Ref: HUM-386) y miembro de la red de Investigación sobre Liderazgo y Mejora Educativa (RILME). Áreas de investigación: Liderazgo y Coordinación docente en Educación Secundaria; Organización Educativa. ORCID ID: 0000-0003-2620-5779.

E-mail: igmartinez@ugr.es

Contact Address: Campus la Cartuja, 18071. Granada (España).

Lina Higueras-Rodríguez. Licenciada en Pedagogía. Máster en Intervención psicopedagógica. Actualmente, es doctoranda en la Facultad de Ciencias de la Educación de la Universidad de Granada, con una vinculación contractual FPU. Miembro del equipo de investigación FORCE y miembro de la red de investigación RILME). Áreas de investigación: Formación docente, Metodologías innovadoras y Liderazgo educativo. ORCID ID: 0000-0002-4458-7339.E-mail: mlina@ugr.es

Contact Address: Campus la Cartuja, 18071. Granada (España). 